\title{
Qualitative behaviour for one-dimensional strongly degenerate parabolic problems
}

\author{
C. MASCIA ${ }^{\dagger}$ \\ Dipartimento di Matematica, Università di Roma "La Sapienza", \\ Piazzale Aldo Moro n. 5, I-00185 Roma, Italy \\ A. PORRETTA \\ Dipartimento di Matematica, Università di Roma "Tor Vergata", \\ Via della Ricerca Scientifica, I-00133 Roma, Italy \\ AND \\ A. TERRACINA ${ }^{\S}$ \\ Dipartimento di Matematica, Università di Roma "La Sapienza", \\ Piazzale Aldo Moro n. 5, I-00185 Roma, Italy
}

[Received 23 December 2004 and in revised form 1 December 2005]

\begin{abstract}
We consider strongly degenerate convection-diffusion equations which mix possible parabolic and hyperbolic behaviour. We prove some qualitative properties of the solutions, in the one-dimensional case. In particular we study the evolution in time of the number of connected components of parabolic and hyperbolic regions and the continuity of the interfaces between the two phases.
\end{abstract}

Keywords: Entropy solutions; strongly degenerate parabolic equations; interface properties; lap number.

\section{Introduction}

We consider the following strongly degenerate parabolic problem:

$$
\begin{cases}u_{t}-b(u)_{x x}+\Phi(u)_{x}=0 & \text { in } R:=(0, L) \times(0, T), \\ u(x, 0)=u_{0}(x) & \text { in }[0, L], \\ u(0, t)=u_{-}(t), \quad u(L, t)=u_{+}(t) & \text { in }[0, T] .\end{cases}
$$

The functions $\Phi$ and $b$ are Lipschitz continuous and represent respectively the convection and the diffusion term of the equation, moreover $b$ is assumed to be nondecreasing. In particular we consider situations in which this function is constant in some intervals of the state variable $u$, so that the equation could degenerate into a hyperbolic conservation law. This kind of equations of mixed parabolic-hyperbolic type appear in different applicative models (see [9, 10, 11, 19]) and have attracted much interest in the last few years. A large number of papers have been devoted to the question of existence and uniqueness of solutions in a suitable formulation. In fact, due

\footnotetext{
${ }^{\dagger}$ E-mail: mascia@mat.uniroma1.it

†E-mail: porretta@mat.uniroma2.it

§E-mail: terracin@mat.uniroma1.it
} 
to the possible hyperbolic character, it is necessary to introduce entropy conditions in the spirit of [25] in order to prove that such problems are well posed. This was done, in much generality and for the multidimensional case, in [14] for homogeneous boundary data, and in [27, 29] for general nonhomogeneous Dirichlet conditions. In all the above works, $L^{1}$-contraction principles were established with different variants of Kružkov's doubling variable technique, while existence was proved with different approaches, respectively through semigroup theory [14], vanishing viscosity limits [27] and numerical schemes [29]. A kinetic approach to approximate strongly degenerate problems was developed in [8, 22], where it is shown that the solution of the hyperbolicparabolic equation can be obtained as a singular limit of a semilinear relaxation system. Numerical approximation schemes are analyzed in [1, 17, 18].

Despite this large amount of references, it seems that very few results are known concerning qualitative properties of solutions to (1) in its full generality. Of course, there is a wide literature on the porous media equation (see e.g. [2, 4, 12, 13, 15, 20, 24] and references therein) as well as on Stefan problems (see [3, 5, 6, 7, 21]). In particular in [3, 7, 21] the effect of nonincreasing and disappearance of the mushy region is studied.

For the analysis of a parabolic-hyperbolic phase transition, we refer to [26] where the author studies a PDE in the class of 1 for a $C^{2}$ function $b$ having exactly one bounded interval of degeneration.

In this paper, we are interested in qualitative properties of the solutions in the one-dimensional case. Our purpose is, roughly speaking, to understand whether and how one can separate the parabolic and hyperbolic regions. More precisely, when the solution $u$ takes values in an interval where $b$ is constant (respectively, where $b$ is strictly increasing) we say that the solution is in a hyperbolic (respectively parabolic) region. We are interested in the evolution in time of the different connected components of parabolic and hyperbolic regions. This can be thought of as a study of the "lap number" of the solution in the spirit of [28]. Basically we prove that the number of connected components of parabolic regions nonincreases in time, so that one can actually separate the evolution of different zones. Precise results are given in Theorem 3.2, the main tool being the maximum principle.

We then look at the interface function $\xi(t)$ between a parabolic and a hyperbolic region, and we give sufficient and necessary conditions for the continuity of $\xi$. Following [20], our arguments use local comparison with super- and subsolutions of travelling wave form.

The paper is organized in three further sections. In the following one, the precise definition of entropy solution of problem (1) is stated, existence and uniqueness results are recalled and moreover a maximum principle for this equation is given, which is our basic tool for studying the structure of the solutions. Section 3 is devoted to the analysis of evolution of connected components of hyperbolic and parabolic phases. Section 4 contains the results on continuity of the interfaces.

\section{Existence and uniqueness}

In this section we state the main known results concerning problem (1). We assume

[H1] $\Phi, b \in \operatorname{Lip}(\mathbb{R}), b$ nondecreasing.

We consider the following definition of entropy solution (see [14, 27, 29]).

NotATion. $\quad H^{ \pm}(s):=\frac{1}{2}(\operatorname{sgn}(s) \pm 1)$ and $[s]_{ \pm}$denotes the positive/negative part of $s$. 
Definition 2.1 Let $T>0$ and $u_{0} \in L^{\infty}(0, L), u_{ \pm} \in L^{\infty}(0, T)$ be given.

A function $u \in L^{\infty}((0, L) \times(0, T))$ is said to be an entropy solution of problem (1) if

(i) (regularity) $b(u) \in L^{2}\left(0, T ; H^{1}(0, L)\right)$ and

$$
b(u)(0, t)=b\left(u_{-}(t)\right) \quad \text { and } \quad b(u)(L, t)=b\left(u_{+}(t)\right) \quad \text { a.e. in }[0, T] ;
$$

(ii) (entropy condition) for any $\psi \in H^{1}((0, L) \times(0, T)), \psi \geqslant 0$, such that

$$
\left.\psi H^{ \pm}\left(u_{-}-k\right)\right|_{\{0\} \times(0, T)}=0 \quad \text { and }\left.\quad \psi H^{ \pm}\left(u_{+}-k\right)\right|_{\{L\} \times(0, T)}=0,
$$

we have

$$
\int_{0}^{L} \int_{0}^{T}\left\{[u-k]_{ \pm} \psi_{t}+K_{x}^{ \pm}(u, k) \psi_{x}\right\} \mathrm{d} x \mathrm{~d} t+\int_{0}^{L}\left[u_{0}-k\right]_{ \pm} \psi \mathrm{d} x \geqslant 0
$$

for any $k \in \mathbb{R}$, where

$$
K_{x}^{ \pm}(u, k):=H^{ \pm}(u-k)\left(\Phi(u)-\Phi(k)-b(u)_{x}\right) .
$$

The following results hold:

TheOREM 2.2 (see [14, 27, 29, 9]) There exists a unique entropy solution to problem (1). Moreover, the solution $u$ is the a.e. limit of solutions $u^{\varepsilon}$ of the parabolic approximating problems

$$
\begin{cases}u_{t}^{\varepsilon}-b_{\varepsilon}\left(u^{\varepsilon}\right)_{x x}+\Phi\left(u^{\varepsilon}\right)_{x}=0 & \text { in } R:=(0, L) \times(0, T), \\ u^{\varepsilon}(x, 0)=u_{0}(x) & \text { in }[0, L], \\ u^{\varepsilon}(0, t)=u_{-}(t), \quad u^{\varepsilon}(L, t)=u_{+}(t) & \text { in }[0, T],\end{cases}
$$

where $b_{\varepsilon}$ is such that $b_{\varepsilon}^{\prime} \geqslant \varepsilon>0$ and $b_{\varepsilon}(\cdot) \rightarrow b(\cdot)$ uniformly on compact sets of $\mathbb{R}$ as $\varepsilon \rightarrow 0^{+}$.

In particular in [9] it is proved that $b(u)$ is continuous in $(0, L) \times(0, T)$ (see also [26], [16]).

As an immediate consequence of Theorem 2.2 and the comparison properties valid for parabolic equations we obtain

Proposition 2.3 Let $u, v$ be solutions of problem (1) with data $u_{0}, v_{0}, u_{ \pm}, v_{ \pm}$, respectively. If $u_{0} \leqslant v_{0}$ a.e. in $(0, L)$ and $u_{-} \leqslant v_{-}, u_{+} \leqslant v_{+}$a.e. in $(0, T)$, then $u \leqslant v$ a.e. in $R$.

Later on, we will use the following consequence of the (weak) maximum principle.

Proposition 2.4 Let $x_{1}, x_{2} \in(0, L), x_{1}<x_{2}$ and let $\gamma$ be a continuous, simple curve, entirely contained in $(0, L) \times(0, t]$ for some $t>0$ with end-points $\left(x_{1}, t\right)$ and $\left(x_{2}, t\right)$. Finally, let $D$ be the domain bounded by $\gamma$ and the segment $\left\{(\xi, t): \xi \in\left[x_{1}, x_{2}\right]\right\}$. Then

$$
\min _{(y, s) \in \gamma} b(u(y, s)) \leqslant b(u(x, t)) \leqslant \max _{(y, s) \in \gamma} b(u(y, s)) \quad \forall(x, t) \in D .
$$

Proof. Let $b_{\varepsilon}$ be a sequence of functions satisfying

(i) $b_{\varepsilon}^{\prime}(s) \geqslant \varepsilon>0$ in $\mathbb{R}$;

(ii) $b_{\varepsilon}(\cdot)$ converges uniformly to $b(\cdot)$ on any compact subset of $\mathbb{R}$ as $\varepsilon \rightarrow 0$. 
Denoting by $u^{\varepsilon}$ the solution of problem (3), since the classical maximum principle applies to the uniformly parabolic equation in $(3)$, we have

$$
\min _{(y, s) \in \gamma} b_{\varepsilon}\left(u^{\varepsilon}(y, s)\right) \leqslant b_{\varepsilon}\left(u^{\varepsilon}(x, t)\right) \leqslant \max _{(y, s) \in \gamma} b_{\varepsilon}\left(u^{\varepsilon}(y, s)\right) \quad \forall(x, t) \in D .
$$

Since $b_{\varepsilon}\left(u^{\varepsilon}\right)$ converges uniformly to $b(u)$ on compact subsets of $R$ (see [23]), the proof is obtained by passing to the limit as $\varepsilon \rightarrow 0$ in (5).

We will mainly consider comparison with solutions that are piecewise smooth (and, additionally, are local travelling wave solutions). Hence it is useful to characterize piecewise regular functions that are entropy solutions of problem (1). To this end, consider $u=u(x, t)$ regular over all $R$ except on a smooth curve. More precisely, assume that there exists a $C^{1}$ curve $\Gamma \subset R$ with normal vector $v=\left(v_{x}, v_{t}\right)$ such that $u \in C^{2}(R \backslash \Gamma)$ (the orientation of $v$ can be chosen arbitrarily). Moreover we assume that the following limits exist and are finite:

$$
\begin{aligned}
u^{ \pm}(x, t) & :=\lim _{\varepsilon \rightarrow 0^{ \pm}} u\left(x+\varepsilon v_{x}, t+\varepsilon v_{t}\right), \\
b(u)_{x}^{ \pm}(x, t) & :=\lim _{\varepsilon \rightarrow 0^{ \pm}} b(u)_{x}\left(x+\varepsilon v_{x}, t+\varepsilon v_{t}\right), \quad(x, t) \in \Gamma .
\end{aligned}
$$

Proposition 2.5 Let $u \in C^{2}(R \backslash \Gamma)$ be such that $(6)$ holds. Then $u$ satisfies the entropy condition 2 for every $\psi \in C_{0}^{1}(R), \psi \geqslant 0$, if and only if

(i) in the sense of distributions, in $R \backslash \Gamma$,

$$
u_{t}(x, t)-b(u(x, t))_{x x}+\Phi(u(x, t))_{x}=0 ;
$$

(ii) (Rankine-Hugoniot condition) in $\Gamma$,

$$
[u] v_{t}+\left[-b(u)_{x}+\Phi(u)\right] \cdot v_{x}=0,
$$

where $[f]=f^{+}-f^{-}$is the jump of $f$ on $\Gamma$;

(iii) in $\Gamma$, for any $k$ between $u^{-}$and $u^{+}$,

$$
[|u-k|] v_{t}+\left[-|b(u)-b(k)|_{x}+\operatorname{sgn}(u-k)(\Phi(u)-\Phi(k))\right] \cdot v_{x} \leqslant 0 .
$$

Conditions (ii) and (iii) can be rewritten as a single one:

(iv) in $\Gamma$, for any $k \in \mathbb{R}$,

$$
[|u-k|] v_{t}+\left[-|b(u)-b(k)|_{x}+\operatorname{sgn}(u-k)(\Phi(u)-\Phi(k))\right] \cdot v_{x} \leqslant 0 .
$$

The proof of Proposition 2.5 is classical and follows the lines of the analogous proof for hyperbolic conservation laws. Here, for completeness, we give a sketch of the proof under the additional smoothness assumptions $b(u) \in C^{2}(R \backslash \Gamma)$ and $\Phi(u) \in C^{1}(R \backslash \Gamma)$.

Proof of Proposition 2.5. Summing the equations with $H_{+}$and $H_{-}$in 2 gives

$$
\int_{\Omega}\left\{|u-k| \psi_{t}+K_{x}(u, k) \psi_{x}\right\} \mathrm{d} x \mathrm{~d} t \geqslant 0
$$


for every $\psi \in C_{0}^{1}(R)$, where

$$
K_{x}(u, k):=\operatorname{sgn}(u-k)\left((\Phi(u)-\Phi(k))-b(u)_{x}\right)=\operatorname{sgn}(u-k)(\Phi(u)-\Phi(k))-|b(u)-b(k)|_{x} .
$$

Suppose that $u$ is an entropy solution of $(1)$. Let $P_{0} \equiv\left(x_{0}, t_{0}\right) \in R \backslash \Gamma$ be fixed, and let $\xi_{n}=$ $\xi_{n}(x, t) \geqslant 0$ be a sequence of functions in $C_{0}^{\infty}(R)$ with support in $B\left(P_{0}, 1 / n\right)$ approximating the Dirac distribution $\delta_{P_{0}}$ concentrated at $P_{0}$.

Choosing $k<-\|u\|_{\infty}\left(\right.$ resp. $\left.k>\|u\|_{\infty}\right)$ in (9) and integrating by parts we obtain

$$
\int_{\Omega}\left\{u_{t}-b(u)_{x x}+\Phi(u)_{x}\right\} \xi_{n} \mathrm{~d} x \mathrm{~d} t \leqslant 0 \quad(\text { resp. } \geqslant 0) .
$$

Passing to the limit as $n \rightarrow \infty$ we get property (i).

Now let $P_{0} \in \Gamma$. For $n$ sufficiently large, $B\left(P_{0}, 1 / n\right)=B_{-} \cup B_{+} \subset R$, where $B_{ \pm}=B \cap\{(x, t)$ : $(x, t)=(y, \tau) \pm \varepsilon v$ for some $(y, \tau) \in \Gamma, \varepsilon>0\}$.

Then the left hand side of 9 ) can be written as the sum of two integrals over $B_{-}$and $B_{+}$. Set $\Gamma_{B}=\Gamma \cap B$. Then, integrating by parts both the terms and applying the divergence theorem, we get

$$
\begin{aligned}
\int_{\Gamma_{B}}\left\{\left|u^{-}-k\right| v_{t}\right. & \left.-\left\{|b(u)-b(k)|_{x}^{-}+\operatorname{sgn}\left(u^{-}-k\right)\left(\Phi\left(u^{-}\right)-\Phi(k)\right)\right\} \cdot v_{x}\right\} \xi_{n} \\
& -\int_{\Gamma_{B}}\left\{\left|u^{+}-k\right| v_{t}-\left\{|b(u)-b(k)|_{x}^{+}+\operatorname{sgn}\left(u^{+}-k\right)\left(\Phi\left(u^{+}\right)-\Phi(k)\right)\right\} \cdot v_{x}\right\} \xi_{n} \geqslant 0
\end{aligned}
$$

(note that $v$ points outward from $B_{-}$and inward from $B_{+}$). Passing to the limit as $n \rightarrow \infty$ yields

$$
\begin{aligned}
\left|u^{+}-k\right| v_{t}-\mid u^{-}- & k \mid v_{t}-\left(|b(u)-b(k)|_{x} \cdot v_{x}\right)^{+}+\left(|b(u)-b(k)|_{x} \cdot v_{x}\right)^{-} \\
& +\operatorname{sgn}\left(u^{+}-k\right)\left(\Phi\left(u^{+}\right)-\Phi(k)\right) \cdot v_{x}-\operatorname{sgn}\left(u^{-}-k\right)\left(\Phi\left(u^{-}\right)-\Phi(k)\right) \cdot v_{x} \leqslant 0
\end{aligned}
$$

for any $k \in \mathbb{R}$. This is condition (iv). Now suppose that $u \in C^{2}(R \backslash \Gamma)$ and satisfies (iv) Then using the divergence theorem in the first member of 9 and reasoning as above, we can prove that this is $\geqslant 0$. In the same way we can see that $u$ is a distributional solution of (1) (weak solution). Using this information we deduce 2 .

Condition (iii) can be interpreted geometrically. Assume $u^{+}<u^{-}$and choose $k \in\left(u^{+}, u^{-}\right)$. Then the above condition (taking into account (ii)) reads

$$
\left(k-u^{+}\right) v_{t}+\left(-\operatorname{sgn}\left(b\left(u^{+}\right)-b(k)\right) b(u)_{x}^{+}+\Phi(k)-\Phi\left(u^{+}\right)\right) \cdot v_{x} \leqslant 0 .
$$

Set $\Phi_{v}(s)=\Phi(s) \cdot v_{x}$ for any $s \in \mathbb{R}$. Then

$$
\Phi_{v}(k) \leqslant \Phi_{v}\left(u^{+}\right)+\operatorname{sgn}\left(b\left(u^{+}\right)-b(k)\right) b(u)_{x}^{+} \cdot v_{x}-\left(k-u^{+}\right) v_{t} .
$$

Similarly,

$$
\Phi_{v}(k) \leqslant \Phi_{v}\left(u^{-}\right)-\operatorname{sgn}\left(b\left(u^{-}\right)-b(k)\right) b(u)_{x}^{-} \cdot v_{x}-\left(k-u^{-}\right) v_{t} .
$$

Then we have

$$
\Phi_{v}(k) \leqslant \Phi_{v}\left(u^{+}\right)-b(u)_{x}^{+} \cdot v_{x}-\left(k-u^{+}\right) v_{t} \quad \forall k \in\left(u^{+}, u^{-}\right),
$$


or, equivalently,

$$
\Phi_{\nu}(k) \leqslant \Phi_{v}\left(u^{-}\right)-b(u)_{x}^{-} \cdot v_{x}-\left(k-u^{-}\right) v_{t} \quad \forall k \in\left(u^{+}, u^{-}\right) .
$$

Note that, letting $k \rightarrow u^{ \pm}$, we deduce that $b(u)_{x}^{ \pm} \cdot v_{x} \leqslant 0$.

Observe that, by the Rankine-Hugoniot condition,

$$
v_{t}=\frac{\Phi_{v}\left(u^{+}\right)-b(u)_{x}^{+} \cdot v_{x}-\Phi_{v}\left(u^{-}\right)+b(u)_{x}^{-} \cdot v_{x}}{u^{+}-u^{-}} .
$$

These conditions can be represented graphically, giving an Oleĭnik-like condition for entropic discontinuity. In this case the straight line is not the graph of the straight line connecting the end

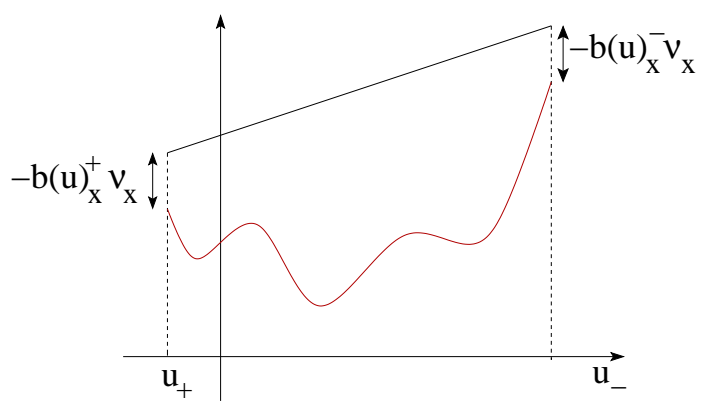

FIG. 1. The geometrical interpretation of the entropy condition.

points of the flux, but it connects some higher points. This means that nonadmissible hyperbolic discontinuities can be admissible in the degenerate parabolic situation thanks to the presence of the diffusive term $b(u)_{x x}$. The interpretation is easy: admissibility of a jump is the analytical translation of compression due to the end states. The presence of diffusion (measured by $-b(u)_{x}$ ) raises the compression strength of the end states, hence admissibility is possible even in cases in which it was not for the corresponding hyperbolic equation.

The case $u_{-}<u_{+}$can be dealt with similarly.

\section{Evolution of parabolic/hyperbolic zones}

Let $u_{0} \in \mathrm{BV}(0, L)$. We assume the additional hypotheses

[H2] $u_{-}(t)=u_{0}(0)=: u_{-} \in \mathbb{R}$ and $u_{+}(t)=u_{0}(L)=: u_{+} \in \mathbb{R}$ for any $t$;

[H3] there exist $I_{i}=\left[\alpha_{i}, \beta_{i}\right], i=1, \ldots, k$, such that $b \in C^{1}\left(\mathbb{R} \backslash\left\{\alpha_{i}, \beta_{i}\right\}\right)$ and

(i) $\beta_{i}<\alpha_{i+1}$ for any $i$,

(ii) $b^{\prime} \equiv 0$ in $I_{i}$ for any $i$,

(iii) $b^{\prime}>0$ in $\mathbb{R} \backslash \bigcup_{i=1}^{k} I_{i}$.

Definition 3.1 The intervals $I_{i}$ are called hyperbolic zones, and the intervals $P_{i}$ where

$$
P_{1}:=\left(-\infty, \alpha_{1}\right], \quad P_{i}:=\left(\beta_{i-1}, \alpha_{i}\right), \quad i=2, \ldots, k, \quad P_{k+1}:=\left(\beta_{k}, \infty\right),
$$

are called parabolic zones. 


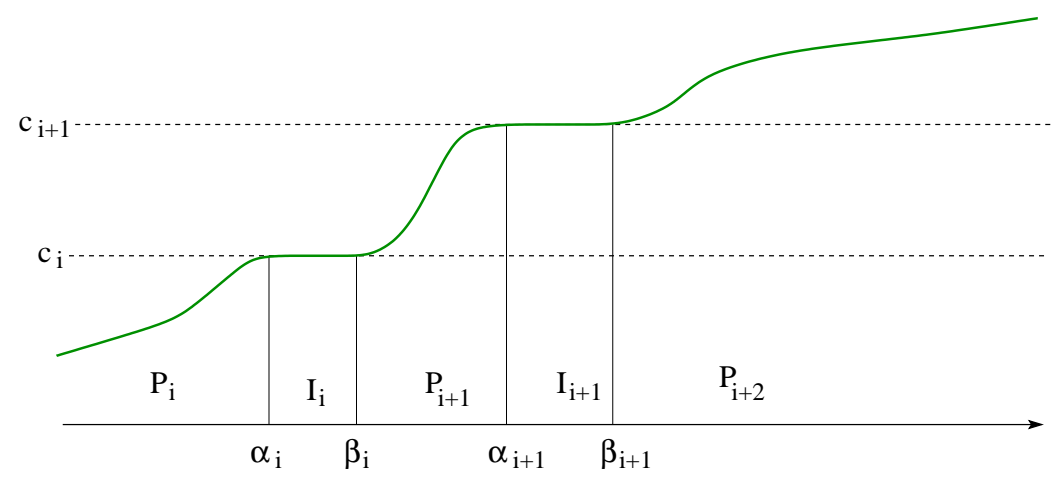

FIG. 2. The graph of the function $b$.

If we set $c_{i}:=b(u) \in \mathbb{R}$ for any $u \in I_{i}$, then for any $u \in P_{i}$ and $i \in\{2, \ldots, k\}, c_{i-1}<b(u)$ $<c_{i}$.

For every $t \in[0, T]$, we introduce the sets

$$
\begin{array}{ll}
N_{i}(t)=\left\{x \in[0, L]: u(x, t) \in P_{i}\right\}, & i=1, \ldots, k+1, \\
H_{i}(t)=\left\{x \in[0, L]: u(x, t) \in I_{i}\right\}, & i=1, \ldots, k .
\end{array}
$$

From the continuity of $b(u(x, t))$ we deduce that $N_{i}(t)$ and $H_{i}(t)$ are unions of disjoint intervals. The intervals of $N_{i}(t)$ are open in $[0, L]$ so they can be of these types: $(\mu, \eta),[0, \eta),(\mu, L],[0, L]$, with $\mu, \eta \in(0, L)$. We denote by $n_{i}(t), h_{i}(t) \in \mathbb{N} \cup\{\infty\}$ the number of connected components of $N_{i}(t)$ and $H_{i}(t)$. Moreover, we let the components be $N_{i, j}(t)\left(j=1, \ldots, n_{i}(t)\right)$ and $H_{i, j}(t)$ $\left(i=1, \ldots, h_{i}(t)\right)$, so that

$$
N_{i}(t)=\bigcup_{j=1}^{n_{i}(t)} N_{i, j}(t) \quad \text { and } \quad H_{i}(t)=\bigcup_{j=1}^{h_{i}(t)} H_{i, j}(t)
$$

Let us state the main result of this section:

Theorem 3.2 Assume [H1]-[H3]. Then the functions $n_{i}=n_{i}(t)$ and $h_{i}=h_{i}(t)$ are nonincreasing in $[0, T]$ for any $i$.

Proof. First we establish the monotonicity of $n_{i}$. Fix $s<t$ and, without loss of generality, assume $n_{i}(s)<\infty$. To simplify notation, we suppress the dependence on the index $i$.

In order to prove Theorem 3.2, we introduce a relation between the sets $S(t):=$ $\left\{N_{1}(t), \ldots, N_{n(t)}(t)\right\}$ and $S(s):=\left\{N_{1}(s), \ldots, N_{n(s)}(s)\right\}$. We recall that every element of $S(t)$ and $S(s)$ is an interval. For $N \in S(t)$ and $\hat{N} \in S(s)$ we write $N \triangleright \hat{N}$ if there exists a continuous function $h(\tau)$ defined in $[s, t]$ such that

(i) $h(s) \in \hat{N}, h(t) \in N$;

(ii) $u(h(\tau), \tau) \in P$ for every $\tau \in[s, t]$,

This could be seen as a "genealogy" relation: $N \triangleright \hat{N}$ means that $N$ is the "father" of $\hat{N}$. As $t$ grows, we go back in the genealogy and, essentially, assign to any interval $N$ its ancestors. The proof of the monotonicity consists in showing that this procedure is uniquely defined. 
As an immediate consequence of the definition, the relation $\triangleright$ is transitive: if $N_{i} \in S\left(t_{i}\right)$ for $i=1,2,3$ with $t_{1} \leqslant t_{2} \leqslant t_{3}$, then

$$
N_{3} \triangleright N_{2}, N_{2} \triangleright N_{1} \Rightarrow N_{3} \triangleright N_{1} .
$$

Moreover, Proposition 2.4 guarantees the validity of the implication

$$
N_{1}, N_{2} \in S(t), N_{1} \triangleright \hat{N}, N_{2} \triangleright \hat{N} \Rightarrow N_{1}=N_{2} .
$$

Indeed, if $N_{1} \triangleright \hat{N}$ and $N_{2} \triangleright \hat{N}$ with $N_{1} \neq N_{2}$, then there exist two functions $h_{1}$ and $h_{2}$ satisfying (i) and (ii) and it is not restrictive to assume $h_{1} \leqslant h_{2}$. As a consequence, it is possible to construct a continuous simple curve $\gamma$ with end-points $\left(h_{1}(t), t\right) \in N_{1}$ and $\left(h_{2}(t), t\right) \in N_{2}$ such that $\left.u\right|_{\gamma} \in P$. Applying Proposition 2.4. we deduce that $u(x, t) \in P$ for any $x \in\left[h_{1}(t), h_{2}(t)\right]$. This means that $N_{1}$ and $N_{2}$ are the same connected component.

Claim. Let $s<t$. Then

$$
\forall N \in S(t), \exists \hat{N} \in S(s) \quad \text { such that } \quad N \triangleright \hat{N} .
$$

Properties (10) and (11) imply that $n$ is nonincreasing. The proof of the Claim is divided into three steps.

STEP 1 Given $N \in S(t)$, let

$$
t^{*}:=\inf \{\tau \in(0, t]: \exists \tilde{N} \in S(\tau) \text { with } N \triangleright \tilde{N}\} .
$$

By continuity of $b(u)$, there exists $\delta>0$ such that for every $\tau \in(t-\delta, t)$ there is $\tilde{N} \in S(\tau)$ such that $N \triangleright \tilde{N}$, hence $t^{*}<t$. We shall show that $t^{*}=0$, thus proving the Claim. If $N=[0, \eta)$ or ( $\mu, L]$, it is possible to choose $h \equiv 0$ or $h \equiv L$, so $t^{*}=0$. Hence, in the following we can consider the case $N \subset(0, L)$.

STEP 2 If $t^{*}>0$, then there exists $\delta>0$ such that for any $\tau \in\left(t^{*}, t^{*}+\delta\right)$ and any $\hat{N} \in S(\tau)$ such that $N \triangleright \hat{N}$ we have $b(u(\xi, \tau))=b(u(\eta, \tau))$ where $\hat{N}=(\xi, \eta)$.

Indeed, if this is not the case, then for any $c \in b(P)$, there exists a sequence $t_{n} \searrow t^{*}$ and $x_{n} \in \tilde{N} \in S\left(t_{n}\right), N \triangleright \tilde{N}$, such that $b\left(u\left(x_{n}, t_{n}\right)\right)=c$. Extracting a subsequence we obtain $x_{n} \rightarrow x^{*}$ and $b\left(u\left(x^{*}, t^{*}\right)\right)=c$. This implies that $\left(x^{*}, t^{*}\right) \in \hat{N} \in S\left(t^{*}\right)$ such that $N \triangleright \hat{N}$. This is a contradiction, since by continuity of $b(u)$ if $t^{*}>0$, then $t^{*}$ does not belong to the set $\{\tau \in(0, t): \exists \tilde{N} \in$ $S(\tau)$ with $N \triangleright \tilde{N}\}$.

STEP $3 t^{*}=0$.

Assume, by contradiction, $t^{*}>0$. By Step 2, without loss of generality, we can assume that $N=(\mu, \eta) \subset(0, L)$ and $b(u(\mu, t))=b(u(\eta, t))=c_{i-1}$. Denote by $D$ the arcwise connected component of the set $\left\{(x, s) \in(0, L) \times(0, t]: b(u)(x, s)>c_{i-1}\right\}$ which intersects $N$.

We claim from Step 2 that $D \subset u^{-1}\left\{P_{i}\right\}$, and moreover using $t^{*}>0$ and $(\mu, \eta) \subset(0, L)$ that $\bar{D} \subset R$.

Indeed, using the fact that $b(u)<c_{i}$ on $N$ and a standard maximum principle argument (see Proposition 2.4) we find that there exists $\sigma>0$ such that $D \cap(t-\sigma, t) \subset u^{-1}\left\{P_{i}\right\}$. Set

$$
\tilde{t}=\inf \left\{s: D \cap(s, t) \subset u^{-1}\left\{P_{i}\right\}\right\} .
$$


Then $\tilde{t}=0$. Indeed, if not there would exist $\tilde{x} \in(0, L)$ such that $(\tilde{x}, \tilde{t}) \in D$ and $b(u(\tilde{x}, \tilde{t}))=c_{i}$; consider the point $x_{0}=\inf \{x:(x, \tilde{x}) \in D \cap[t=\tilde{t}]\}$; by continuity of $b(u)$ we have $x_{0}<\tilde{x}$, and either $b\left(u\left(x_{0}, \tilde{t}\right)\right)=c_{i-1}$ or $x_{0}=0$. In the first case, since $D \cap(\tilde{t}, t) \subset u^{-1}\left\{P_{i}\right\}$, this would give rise to an interval $\tilde{N}=\left(x_{0}, \tilde{x}\right)$ such that $N \triangleright \tilde{N}$ but $b(u)$ does not have constant value on $\partial \tilde{N}$, contradicting Step 2. In the second case, using the boundary $x=0$, the segment $(0, \tilde{x})$ and an arc in $D$, a simple application of Proposition 2.4 will contradict the fact that $N \subset(0, L)$ (i.e. $N$ does not include the boundary points).

Therefore $D \subset u^{-1}\left\{P_{i}\right\}$; moreover, similar arguments and the facts that $t^{*}>0$ and $N \subset(0, L)$ prove that $\bar{D} \subset R$.

For any $\varepsilon>0$ let $u^{\varepsilon}$ be a solution of (3) such that

$$
\left|b_{\varepsilon}\left(u^{\varepsilon}\right)-b(u)\right|<\varepsilon \quad \text { in } \bar{D} .
$$

Then there exists an interval $I \subset(\mu, \eta)$ such that $b_{\varepsilon}\left(u^{\varepsilon}\right) \geqslant c_{i-1}+\varepsilon$ on $I$ (for any $\varepsilon$ small enough). Denote by $D_{\varepsilon}$ the arcwise connected component of the set $\left\{(x, s) \in(0, L) \times(0, t]: b_{\varepsilon}\left(u^{\varepsilon}\right)(x, s)>\right.$ $\left.c_{i-1}+\varepsilon\right\}$ which contains $I$. Clearly, $b_{\varepsilon}\left(u^{\varepsilon}\right)=c_{i-1}+\varepsilon$ on $\partial D_{\varepsilon}$. Moreover, $D_{\varepsilon} \subset D$ and we deduce that $\partial D_{\varepsilon}$ does not intersect the line $t=0$, nor the boundary $x=0, x=L$.

We now apply to the parabolic equation $\left(3\right.$ the strong maximum principle: if $\max _{\bar{D}_{\varepsilon}} b_{\varepsilon}\left(u^{\varepsilon}\right)(x, s)$ is assumed at a point $\left(x_{0}, t_{0}\right) \in D_{\varepsilon}$ or at $s=t$, we deduce that $b_{\varepsilon}\left(u^{\varepsilon}\right)(x, s)$ is constant on the whole line $s=t_{0}$ or $s=t$; since $\partial D_{\varepsilon} \cap\left\{t=t_{0}\right\} \neq \emptyset$ and $b_{\varepsilon}\left(u^{\varepsilon}\right)=c_{i-1}+\varepsilon$ on $\partial D_{\varepsilon}$ we conclude that $\max _{\bar{D}_{\varepsilon}} b_{\varepsilon}\left(u^{\varepsilon}\right)=c_{i-1}+\varepsilon$, and in particular $b_{\varepsilon}\left(u^{\varepsilon}\right) \leqslant c_{i-1}+\varepsilon$ on the segment $I \subset(\mu, \eta)$. We deduce that $b(u)<c_{i-1}+2 \varepsilon$ on $I$. Letting $\varepsilon \rightarrow 0$ we find $b(u)=c_{i-1}$ on $I \subset N$, which is a contradiction.

This implies $t^{*}=0$ and the Claim is proved.

It remains to prove that also the functions $h_{i}$ are nonincreasing. Loosely speaking, we need to prove the hyperbolic version of properties (10) and (11) just proved for the parabolic regions.

Let us drop again the index $i$. Let $t>0$ be such that there exist two connected components $H_{1}=\left[\eta_{1}, \xi_{1}\right]$ and $H_{2}=\left[\eta_{2}, \xi_{2}\right]$ of the same hyperbolic region with $\eta_{1} \leqslant \xi_{1}<\eta_{2} \leqslant \xi_{2}$. Then there exists $N \in\left(\xi_{1}, \eta_{2}\right)$ belonging to some parabolic region $P$. By the result proved for the parabolic regions, $N$ is such that for any $s<t$ there exists $\hat{N}$ with $N \triangleright \hat{N}$. Hence there cannot exist any hyperbolic connected component $\hat{H}$ at time $s$ such that $H_{1} \triangleright \hat{H}$ and $H_{2} \triangleright \hat{H}$. This proves the analogue of $(10)$.

Similarly, by the maximum principle and the results already proved for parabolic regions, it can be proved that also (11) holds for the hyperbolic intervals.

\section{Continuity of the interfaces}

In this section we are interested in the study of the interface curves between parabolic and hyperbolic regions.

For fixed $i$, consider a connected component of $N_{i}(0)=\left\{x: b\left(u_{0}(x)\right) \in\left(c_{i-1}, c_{i}\right)\right\}$, say $N_{i, \ell}(0)=\left(\xi_{i, \ell}(0), \zeta_{i, \ell}(0)\right)$. To simplify notation we can assume that $u_{-}$and $u_{+}$are in hyperbolic regions so that the connected components are open. Set

$$
T^{*}:=\sup \left\{t: \exists N(t) \triangleright N_{i \ell}(0)\right\},
$$

where $\triangleright$ is as in the previous section. By continuity of $b(u), T^{*}>0$. Moreover $N(t)$, whenever it exists, is unique (see the proof of Theorem 3.2); we denote it by $N(t)=\left(\xi_{i, \ell}(t), \zeta_{i, \ell}(t)\right)$. Note that if $\xi_{i, \ell}, \zeta_{i, \ell}$ are defined at time $t$, then they are also defined at time $s$ for any $s \in(0, t)$. 


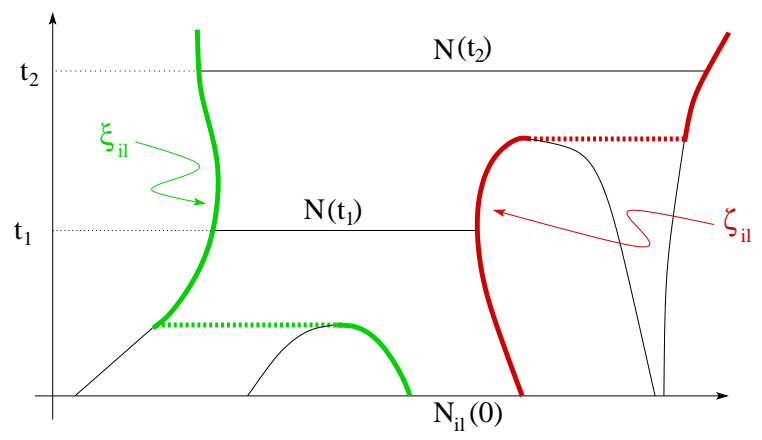

FIG. 3. The sets $N_{i \ell}(0), N(t)$ and the interfaces $\xi_{i, \ell}(t), \zeta_{i, \ell}(t)$

For any $i_{1}, i_{2}, \ell, m$ and for any $t>0, \xi_{i_{1}, \ell}(0) \leqslant \xi_{i_{2}, m}(0)$ and $\zeta_{i_{1}, \ell}(0) \leqslant \zeta_{i_{2}, m}(0)$ imply $\xi_{i_{1}, \ell}(t) \leqslant$ $\xi_{i_{2}, m}(t)$ and $\zeta_{i_{1}, \ell}(t) \leqslant \zeta_{i_{2}, m}(t)$, with $<$ in place of $\leqslant$ if $i_{1} \neq i_{2}$.

Moreover, since parabolic intervals can coalesce in finite time, it can happen that $\xi_{i, \ell}(t)=$ $\xi_{i, m}(t)$ for some $t$ (here $\ell \neq m$ ). Similarly for $\zeta_{i, \ell}$. These functions $\xi_{i, \ell}, \zeta_{i, \ell}$ represent the interface curves between hyperbolic and parabolic regions. In the following, for simplicity we drop the indices $i, j$ and we consider the left interface $\xi(t), t<T^{*}$.

For simplicity, we consider initial/boundary data with image contained in two zones: a hyperbolic one and a parabolic one. More precisely, we consider the case

$$
\begin{gathered}
I=[\alpha, \beta], \quad P=(\beta, \gamma), \quad u\left(\xi(t)^{+}, t\right)=\beta, \\
b(\alpha)=b(\beta)=c, \quad b(\gamma)=d,
\end{gathered}
$$

with $u_{0}(x), u_{ \pm} \subset[\alpha, \gamma)$ for any $x \in(0, L)$.

Proposition 4.1 For any $t_{0}>0$, we have $\lim _{t \rightarrow t_{0}^{-}} \xi(t)=\xi\left(t_{0}\right)$.

Proof. STEP 1 We have

$$
\limsup _{t \rightarrow t_{0}^{-}} \xi(t) \leqslant \xi\left(t_{0}\right) \quad \text { and } \quad \liminf _{t \rightarrow t_{0}^{-}} \zeta(t) \geqslant \zeta\left(t_{0}\right)
$$

Indeed, by continuity of $b(u)$, any point $\left(x, t_{0}\right)$ with $x \in\left(\xi\left(t_{0}\right), \zeta\left(t_{0}\right)\right)$ has a neighbourhood entirely contained in the parabolic region, and therefore there exists $\delta>0$ such that $\xi(\tau) \leqslant x \leqslant$ $\zeta(\tau)$ for any $\tau \in\left(t_{0}-\delta, t_{0}\right)$. This implies

$$
\limsup _{t \rightarrow t_{0}^{-}} \xi(t) \leqslant x \leqslant \liminf _{t \rightarrow t_{0}^{-}} \zeta(t) .
$$

Since $x$ is arbitrarily chosen in $\left(\xi\left(t_{0}\right), \zeta\left(t_{0}\right)\right)$, this implies the claim.

STEP 2 If there exist $t_{n} \nearrow t_{0}$ as $n \rightarrow \infty$ such that

$$
\lim _{n \rightarrow \infty} \xi\left(t_{n}\right)=\ell<\xi\left(t_{0}\right),
$$

then $u\left(x, t_{0}\right)=\beta$ for any $x \in\left(\ell, \xi\left(t_{0}\right)\right)$. 
Indeed, by Step $1, \liminf _{n \rightarrow \infty} \zeta\left(t_{n}\right) \geqslant \zeta\left(t_{0}\right) \geqslant \xi\left(t_{0}\right)$. Thus, by continuity, $b\left(u\left(x, t_{0}\right)\right) \in[c, d]$ for any $x \in\left(\ell, \xi\left(t_{0}\right)\right)$. If $b\left(u\left(x_{0}, t_{0}\right)\right) \in(c, d)$ for some $x_{0} \in\left(\ell, \xi\left(t_{0}\right)\right)$, then there exists $\delta>0$ such that $b\left(u\left(x_{0}, t\right)\right) \in(c, d)$ for every $t \in\left(t_{0}-\delta, t_{0}\right)$ and $x_{0}$ is in $\left(\xi\left(t_{n}\right), \zeta\left(t_{n}\right)\right)$ for any sufficiently large $n$. This implies that $x_{0} \in\left(\xi\left(t_{0}\right), \zeta\left(t_{0}\right)\right)$, which is a contradiction. Hence $b\left(u\left(x_{0}, t_{0}\right)\right) \in\{c, d\}$.

Finally, $b\left(u\left(x_{0}, t_{0}\right)\right) \neq d$, since this would imply the existence of $x_{1} \in\left(x_{0}, \xi\left(t_{0}\right)\right)$ with $b\left(u\left(x_{1}, t_{0}\right)\right) \in(c, d)$. Hence $b\left(u\left(x_{0}, t_{0}\right)\right)=c$ for any $x \in\left(\ell, \xi\left(t_{0}\right)\right)$. Since $u \in[\beta, \gamma]$ for $x \in\left(\ell, \xi\left(t_{0}\right)\right)$, we conclude that $u\left(x, t_{0}\right)=\beta$ for any $x \in\left(\ell, \xi\left(t_{0}\right)\right)$.

STEP 3 Construction of (local) travelling waves. Let $t_{n} \nearrow t_{0}$ as $n \rightarrow \infty$ be such that (12) holds and let $c>\operatorname{Lip} \Phi$. Then there exist $\varepsilon>0$ and $n_{0} \in \mathbb{N}$ such that

$$
\begin{aligned}
& \ell+2 \varepsilon+\int_{\alpha-\varepsilon}^{\alpha} \frac{b^{\prime}(s)}{\Phi(s)-c s-(\Phi(\beta)-c \beta)} \mathrm{d} s<\xi\left(t_{0}\right), \\
& u(\ell+\varepsilon, t) \geqslant \alpha-\varepsilon \quad \forall t \in\left[t_{n_{0}}, t_{0}\right],
\end{aligned}
$$

the latter being a consequence of Step 2 and of continuity of $b(u)$. Moreover, without loss of generality, we can assume $\xi\left(t_{n}\right)<\ell+\varepsilon$ and $c\left(t_{0}-t_{n}\right)<\varepsilon$ for any $n \geqslant n_{0}$.

Let $\bar{x}>\xi\left(t_{0}\right)$ and $\mu>\beta$ be such that $u(\bar{x}, t) \geqslant \mu$ for every $t \geqslant t_{n_{0}}$. We define a travelling wave solution $U_{n}$ in the domain $(\ell+\varepsilon, \bar{x}) \times\left(t_{n}, t_{0}\right)$. To this end, set $\tilde{\mu}_{n}:=\inf \left\{u\left(x, t_{n}\right): x \in(\ell+\varepsilon, \bar{x})\right\}$ and define

$$
\mu_{n}:=\min \left(\tilde{\mu}_{n}, \mu\right) \quad \text { and } \quad \gamma_{n}:=\Phi\left(\mu_{n}\right)-c \mu_{n} .
$$

Since $\xi\left(t_{n}\right)<\ell+\varepsilon$, we have $\mu_{n}>\beta$. Moreover, $\Phi(s)-c s$ is a decreasing function, hence $\Phi(s)-c s>\gamma_{n}$ for any $s \in\left(\alpha-\varepsilon, \mu_{n}\right)$. Therefore we can set

$$
x_{n}:=\ell+\varepsilon+\int_{\alpha-\varepsilon}^{\alpha} \frac{b^{\prime}(s)}{\Phi(s)-c s-\gamma_{n}} \mathrm{~d} s .
$$

The function $U_{n}=U_{n}(x-c t)$ implicitly defined by

$$
\begin{cases}\int_{\beta}^{U_{n}} \frac{b^{\prime}(s)}{\Phi(s)-c s-\gamma_{n}} \mathrm{~d} s=x-x_{n}-c\left(t-t_{n}\right) & \text { if } x \geqslant x_{n}+c\left(t-t_{n}\right), \\ \int_{\alpha}^{U_{n}} \frac{b^{\prime}(s)}{\Phi(s)-c s-\gamma_{n}} \mathrm{~d} s=x-x_{n}-c\left(t-t_{n}\right) & \text { if } x<x_{n}+c\left(t-t_{n}\right),\end{cases}
$$

is an increasing travelling wave with jump discontinuity at $x=x_{n}+c\left(t-t_{n}\right)$ from $\alpha$ to $\beta$. Moreover, $U_{n}$ satisfies both the Rankine-Hugoniot and entropy condition (7)-(8) (since $\Phi(s)-c s>\gamma_{n}$ for $s \in(\alpha, \beta)$ ), and, thanks to Proposition 2.5, it is an entropy solution. By definition of $x_{n}$,

$$
U_{n}(\ell+\varepsilon, t) \leqslant \alpha-\varepsilon \leqslant u(\ell+\varepsilon, t) \quad \forall t \in\left(t_{n}, t_{0}\right),
$$

and since $\int_{\beta}^{\mu_{n}} \frac{b^{\prime}(s)}{\Phi(s)-c s-\gamma_{n}} \mathrm{~d} s=\infty$,

$$
U_{n}<\mu_{n} \leqslant \min \left\{u\left(x, t_{n}\right), u(\bar{x}, t): x \in(\ell+\varepsilon, \bar{x}), t \in\left(t_{n}, t_{0}\right)\right\} .
$$

We conclude that $U_{n} \leqslant u$ on the boundary of the domain $(\ell+\varepsilon, \bar{x}) \times\left(t_{n}, t_{0}\right)$, and, by the comparison principle (Proposition 2.4,$U_{n} \leqslant u$ in $(\ell+\varepsilon, \bar{x}) \times\left(t_{n}, t_{0}\right)$. In particular, we get

$$
u(x, t)>\beta \quad \forall(x, t) \text { such that } x>x_{n}+c\left(t-t_{n}\right) .
$$


By [13] and since $\Phi(s)-c s$ is decreasing we can estimate

$$
\begin{aligned}
x_{n}+c\left(t_{0}-t_{n}\right) & <\ell+\varepsilon+\int_{\alpha-\varepsilon}^{\alpha} \frac{b^{\prime}(s)}{\Phi(s)-c s-\gamma_{n}} \mathrm{~d} s+\varepsilon \\
& \leqslant \ell+2 \varepsilon+\int_{\alpha-\varepsilon}^{\alpha} \frac{b^{\prime}(s)}{\Phi(s)-c s-(\Phi(\beta)-c \beta)} \mathrm{d} s<\xi\left(t_{0}\right),
\end{aligned}
$$

so we conclude that $u\left(\xi\left(t_{0}\right), t_{0}\right)>\beta$, getting a contradiction. Thus (12) cannot hold true, and therefore $\lim _{t_{n} \nearrow t_{0}} \xi\left(t_{n}\right)=\xi\left(t_{0}\right)$.

The same argument as for the previous proposition can be used to prove the existence of the limit from above.

Proposition 4.2 For any $t_{0}>0$, the limit $\lim _{t \rightarrow t_{0}^{+}} \xi(t)$ exists.

Proof. Assume by contradiction that there exist two decreasing sequences $\left\{t_{n}\right\}$ and $\left\{\tau_{n}\right\}$ such that $t_{n}<\tau_{n}$ and

$$
\lim _{t_{n} \rightarrow t_{0}^{+}} \xi\left(t_{n}\right)=\liminf _{t \rightarrow t_{0}^{+}} \xi(t)=: \ell<m:=\limsup _{t \rightarrow t_{0}^{+}} \xi(t)=\lim _{\tau_{n} \rightarrow t_{0}^{+}} \xi\left(\tau_{n}\right)
$$

Since, as in Step 2 in the proof of Proposition 4.1, we have $b(u)(x, t)=c$ for any $x \in(l, m)$, there exists $n_{0}$ such that $u(l+\varepsilon, t)>\alpha-\varepsilon$ for any $t \in\left[t_{0}, \tau_{n_{0}}\right)$. Similarly there exist $\bar{x}>m$ and $\mu>\beta$ such that $u(\bar{x}, t)>\mu$ for every $t \in\left[t_{0}, \tau_{n_{0}}\right)$. We can use the same travelling wave solution $U_{n}$ of Proposition 4.1 in the domain $(l+\varepsilon, \bar{x}) \times\left(t_{n}, \tau_{n_{0}}\right)$, once we choose $\mu_{n}$ such that $u\left(x, t_{n}\right) \geqslant \mu_{n}>\beta$ for every $x \in(l+\varepsilon, \bar{x})$. Since $m>\ell$ we can take $\varepsilon$ such that $c\left(\tau_{n}-t_{n}\right)<\varepsilon$ and

$$
\ell+2 \varepsilon+\int_{\alpha-\varepsilon}^{\alpha} \frac{b^{\prime}(s)}{\Phi(s)-c s-(\Phi(\beta)-c \beta)} \mathrm{d} s<m .
$$

Comparing $u$ and $U_{n}$ we find that $u(x, t)>\beta$ if

$$
x>c\left(t-t_{n}\right)+\int_{\alpha-\varepsilon}^{\alpha} \frac{b^{\prime}(s)}{\Phi(s)-c s-(\Phi(\beta)-c \beta)} \mathrm{d} s+l+\varepsilon .
$$

Since $\xi\left(\tau_{n}\right) \rightarrow m$, the previous inequality is satisfied by $x=\xi\left(\tau_{n}\right), t=\tau_{n}$ as soon as $n$ is large enough, hence we get $u\left(\xi\left(\tau_{n}\right), \tau_{n}\right)>\beta$, which is a contradiction.

The continuity of $\xi(t)$ at $t=t_{0}$ will depend on the behaviour of $b(s)$ at $s=\beta$ and on the situation occurring to the left of $\xi\left(t_{0}\right)$.

Proposition 4.3 Let $b(s)$ satisfy

$$
\int_{\beta}^{\beta+\varepsilon} \frac{b^{\prime}(s)}{s-\beta} \mathrm{d} s=\infty \quad \text { for some } \varepsilon>0 .
$$

Then for any $t_{0} \geqslant 0, \xi(t)$ is discontinuous at $t=t_{0}$ if and only if there exists $x_{0}<\xi\left(t_{0}\right)$ such that $u\left(x, t_{0}\right) \geqslant \beta$ for every $x \in\left(x_{0}, \xi\left(t_{0}\right)\right)$. 
Proof. The necessity is trivial. Indeed, if $\xi(t)$ is discontinuous at $t=t_{0}$, then there exist a point $x_{0}<\xi\left(t_{0}\right)$ and a sequence $t_{n} \rightarrow t_{0}^{+}$such that $\xi\left(t_{n}\right) \rightarrow x_{0}$. Since $u\left(x, t_{n}\right) \in(\beta, \gamma)$ for any $x \in$ $\left(x_{0}, \xi\left(t_{0}\right)\right)$ and $n$ large enough, by continuity of $b(u)$ and since $b^{\prime}(s)>0$ for $s \in(\beta, \gamma)$ we deduce that $u\left(x, t_{0}\right) \geqslant \beta$ for $x \in\left(x_{0}, \xi\left(t_{0}\right)\right)$.

Assume conversely that $u\left(x, t_{0}\right) \geqslant \beta$ for $x \in\left(x_{0}, \xi\left(t_{0}\right)\right)$; let $\xi_{0}>\xi\left(t_{0}\right)$ be such that $u\left(\xi_{0}, t\right)>$ $\mu>\beta$ for $t$ sufficiently close to $t_{0}$. Since for $t$ close to $t_{0}$ we have $u\left(x_{0}, t\right)>\alpha-\varepsilon$, we can compare $u$ in $\left(x_{0}, \xi_{0}\right) \times\left(t_{0}, t\right)$ with a travelling wave solution $U$ taking values between $\alpha-\varepsilon$ and $\beta$, and conclude that there exist $\delta>0$ and $x_{1} \in\left(x_{0}, \xi\left(t_{0}\right)\right)$ such that

$$
u\left(x_{1}, t\right) \geqslant \beta \quad \forall t \in\left[t_{0}, t_{0}+\delta\right) .
$$

Without loss of generality we can assume that $\xi_{0}-\xi\left(t_{0}\right)<\xi\left(t_{0}\right)-x_{1}$. Let now $t_{n}$ be a sequence converging to $t_{0}$ and let $c_{n}=-2\left(\xi_{0}-\xi\left(t_{0}\right)\right) /\left(t_{n}-t_{0}\right)$. Note that $c_{n} \rightarrow-\infty$, so that the function $\Phi(s)-c_{n} s$ is increasing for $n$ large. Since by assumption (14),

$$
\lim _{\gamma \rightarrow 0} \int_{\beta}^{\mu} \frac{b^{\prime}(s)}{\Phi(s)-c_{n} s-\left(\Phi(\beta)-c_{n} \beta\right)+\gamma} \mathrm{d} s=\infty
$$

for every $n$ there exists a constant $\gamma_{n}>0$ such that

$$
2\left(\xi_{0}-\xi\left(t_{0}\right)\right)<\int_{\beta}^{\mu} \frac{b^{\prime}(s)}{\Phi(s)-c_{n} s-\left(\Phi(\beta)-c_{n} \beta\right)+\gamma_{n}} \mathrm{~d} s \quad \forall n .
$$

Define a travelling wave $U_{n}=U_{n}\left(x-c_{n} t\right)$ by

$$
\begin{cases}\int_{\beta}^{U_{n}} \frac{b^{\prime}(s)}{\Phi(s)-c_{n} s-\left(\Phi(\beta)-c_{n} \beta\right)+\gamma_{n}} \mathrm{~d} s=x-c_{n} t-\xi_{0}+c_{n} t_{0} & \text { if } x \geqslant \xi_{0}+c_{n}\left(t-t_{0}\right), \\ U_{n}=\beta^{*} & \text { if } x<\xi_{0}+c_{n}\left(t-t_{0}\right),\end{cases}
$$

where $\Phi\left(\beta^{*}\right)-c_{n} \beta^{*}=\Phi(\beta)-c_{n} \beta-\gamma_{n}$. Observe that since $\Phi(s)-c_{n} s$ is increasing we have $\beta^{*} \leqslant \beta$ and $U_{n}$ is nondecreasing. The travelling wave $U_{n}$ satisfies conditions $(7)-(8)$, hence it is an entropy solution.

Since $x_{1}-c_{n}\left(t_{n}-t_{0}\right)=x_{1}+2\left(\xi_{0}-\xi\left(t_{0}\right)\right)<\xi_{0}, U_{n}\left(x_{1}-c_{n} t\right)=\beta^{*}$ for any $t \in\left[t_{0}, t_{n}\right)$. Moreover, since for $t \leqslant t_{n}$,

$$
-c_{n}\left(t-t_{0}\right) \leqslant-c_{n}\left(t_{n}-t_{0}\right)=2\left(\xi_{0}-\xi\left(t_{0}\right)\right)<\int_{\beta}^{\mu} \frac{b^{\prime}(s)}{\Phi(s)-c s-\left(\Phi(\beta)-c_{n} \beta\right)+\gamma_{n}} \mathrm{~d} s,
$$

we also have $U_{n}\left(\xi_{0}, t\right) \leqslant \mu \leqslant u\left(\xi_{0}, t\right)$ for any $t \in\left[t_{0}, t_{n}\right)$. Clearly at $t=t_{0}$ we have $U_{n}\left(x, t_{0}\right)=$ $\beta^{*} \leqslant u\left(x, t_{0}\right)$ for all $x \in\left(x_{1}, \xi_{0}\right)$. Therefore $U_{n} \leqslant u$ on the parabolic boundary of the rectangle $\left(x_{1}, \xi_{0}\right) \times\left[t_{0}, t_{n}\right)$, and, by the comparison principle, $U_{n} \leqslant u$ inside it. In particular

$$
u(x, t)>\beta \quad \forall(x, t) \text { such that } x>c_{n}\left(t-t_{0}\right)+\xi_{0} .
$$

If $\xi\left(t_{n}\right) \rightarrow \xi\left(t_{0}\right)$, since $c_{n}\left(t_{n}-t_{0}\right)+\xi_{0}=\xi_{0}-2\left(\xi_{0}-\xi\left(t_{0}\right)\right)<\xi\left(t_{0}\right)$, we have $u\left(\xi\left(t_{n}\right), t_{n}\right)>\beta$ for $n$ large enough, which contradicts the definition of $\xi\left(t_{n}\right)$. Hence $\xi(t)$ is discontinuous at $t=t_{0}$. 
Proposition 4.3 gives necessary and sufficient conditions for the formation of a discontinuity at time $t_{0}$. In specific situations, it is possible to deduce that the necessary condition is not satisfied for any positive time, so that the continuity of $\xi$ is ensured. We give an example of such a situation.

EXAmple. Assume additionally $b^{\prime}(\beta+)=b_{0}>0$ and $\Phi \in C^{2}$. Consider the Cauchy problem given by the initial data

$$
u_{0}(x)= \begin{cases}\alpha, & x<0 \text { or } \ell_{1}<x<\ell_{1}+\ell_{2} \\ \beta, & 0<x<\ell_{1} \\ v_{0}(x), & x>\ell_{1}+\ell_{2}\end{cases}
$$

where $v_{0}(x)>\beta$ for any $x$. For which $\ell_{1}, \ell_{2}$ and $v_{0}$ is the interface $\xi$ continuous for any time $t$ ?

The interface curve $\xi$ starts from the point $\ell_{1}+\ell_{2}$. A discontinuity of $\xi$ at some positive time $t_{0}$ is possible if and only if $u\left(x, t_{0}\right)=\beta$ for any $x \in\left(\xi\left(t_{0}\right)-\delta, \xi\left(t_{0}\right)\right)$. Hence if the interval in which the solution $u$ takes the value $\beta$ disappears before intersecting the interface, the function $\xi$ is continuous for any $t$.

The interval in which the solution $u$ takes the value $\beta$ is obtained by solving two Riemann problems. This interval is bounded by the straight lines

$$
x=\Phi_{\text {conv }}^{\prime}(\beta) t, \quad x=\ell_{1}+\Phi_{\text {conc }}^{\prime}(\beta) t
$$

where $\Phi_{\text {conv }}$ and $\Phi_{\text {conc }}$ denote respectively the convex and concave envelopes of $\Phi$ in $[\alpha, \beta]$. Hence the timelife $T$ of $\beta$ is

$$
T:=\frac{\ell_{1}}{\Phi_{\text {conv }}^{\prime}(\beta)-\Phi_{\text {conc }}^{\prime}(\beta)},
$$

if $\Phi$ is not affine in $[\alpha, \beta]$; if $\Phi$ is affine in $[\alpha, \beta]$, then $T:=\infty$.

Next we need to estimate the speed of propagation of the interface $\xi$. Here we proceed formally, the rigorous proof can be obtained by approximating the degenerate parabolic equation with a uniformly parabolic one.

It is known that

$$
\xi^{\prime}(t)=\frac{\Phi(\beta)-\Phi(\alpha)}{\beta-\alpha}-\frac{b(u)_{x}^{+}}{\beta-\alpha} .
$$

The quantity $w=b(u)_{x}$ satisfies

$$
w_{t}=b^{\prime}(u) w_{x x}+\left[b^{\prime}(u)_{x}-\Phi^{\prime}(u)\right] w_{x}-\frac{\Phi^{\prime \prime}(u)}{b^{\prime}(u)} w^{2} .
$$

Set $\mu:=\sup _{u>\beta}\left[-\Phi^{\prime \prime}(u) / b^{\prime}(u)\right]$. In the following, we consider the case $\mu>0$, the other case being easier due to a rarefaction effect in the case of $\Phi$ convex. By comparison, the function $\sup _{x}|w|$ satisfies

$$
\sup _{x}|w|(t) \leqslant \frac{W_{0}}{1-\mu W_{0} t} \quad \forall t \in\left[0,1 / \mu W_{0}\right) \quad \text { where } W_{0}:=\sup \left|w_{0}\right| .
$$

Therefore

$$
\xi^{\prime}(t) \geqslant \frac{\Phi(\beta)-\Phi(\alpha)}{\beta-\alpha}-\frac{W_{0}}{(\beta-\alpha)\left(1-\mu W_{0} t\right)},
$$

so that

$$
\xi(t) \geqslant \tilde{\xi}(t):=\ell_{1}+\ell_{2}+\frac{\Phi(\beta)-\Phi(\alpha)}{\beta-\alpha} t+\frac{1}{\mu(\beta-\alpha)} \ln \left(1-\mu W_{0} t\right) .
$$


Thus, if $T<1 / \mu W_{0}$ and $\Phi_{\text {conv }}^{\prime}(\beta) T<\tilde{\xi}(T)$, the interval in which the solution takes the value $\beta$ disappears before intersecting the interface $\xi$, which, as a consequence, is continuous. The second condition can be rewritten as

$$
\frac{\frac{\Phi(\beta)-\Phi(\alpha)}{\beta-\alpha}-\Phi_{\text {conc }}^{\prime}(\beta)}{\Phi_{\text {conv }}^{\prime}(\beta)-\Phi_{\text {conc }}^{\prime}(\beta)} \ell_{1}+\ell_{2}+\frac{1}{\mu(\beta-\alpha)} \ln \left(1-\frac{\mu W_{0} \ell_{1}}{\Phi_{\text {conv }}^{\prime}(\beta)-\Phi_{\text {conc }}^{\prime}(\beta)}\right)>0 .
$$

The first term is positive, hence the relation is satisfied if

$$
\ell_{2}+\frac{1}{\mu(\beta-\alpha)} \ln \left(1-\frac{\mu W_{0} \ell_{1}}{\Phi_{\mathrm{conv}}^{\prime}(\beta)-\Phi_{\mathrm{conc}}^{\prime}(\beta)}\right)>0,
$$

which is equivalent to

$$
W_{0} \ell_{1}<\frac{1}{\mu}\left(\Phi_{\text {conv }}^{\prime}(\beta)-\Phi_{\text {conc }}^{\prime}(\beta)\right)\left(1-e^{-\mu(\beta-\alpha) \ell_{2}}\right) .
$$

Summarizing, the following condition guarantees the continuity of the interface $\xi$ :

$$
\left|b\left(v_{0}\right)_{x}(x)\right| \ell_{1}<\frac{1}{\mu}\left(\Phi_{\text {conv }}^{\prime}(\beta)-\Phi_{\text {conc }}^{\prime}(\beta)\right) \quad \forall x .
$$

By the comparison principle, (15) guarantees the continuity of $\xi$ also for initial data less than or equal to $u_{0}$.

In case $b(s)$ has finite speed of propagation, the following result holds.

Proposition 4.4 Let $b(s)$ satisfy

$$
\int_{\beta}^{\beta+\varepsilon} \frac{b^{\prime}(s)}{s-\beta} \mathrm{d} s<\infty \quad \text { for some } \varepsilon>0 .
$$

If $u\left(x, t_{0}\right) \leqslant \beta$ for $x \in\left(\xi\left(t_{0}\right)-\delta, \xi\left(t_{0}\right)\right)$ for some $\delta>0$, then $\xi(t)$ is continuous at $t_{0}$.

Proof. Assume by contradiction that $\lim _{t \rightarrow t_{0}^{+}} \xi(t)<\xi\left(t_{0}\right)$ (this limit exists by Proposition 4.2). Then there exists $l<\xi\left(t_{0}\right)$ such that $u\left(x, t_{0}\right)=\beta$ if $x \in\left(l, \xi\left(t_{0}\right)\right)$. Let $c>\operatorname{Lip} \Phi$. By continuity of $b(u)$, there exist $\sigma, \delta>0$ such that

$$
u\left(\xi\left(t_{0}\right), t\right)<\beta+\sigma, \quad u(l, t)<\beta+\sigma \quad \forall t \in\left[t_{0}, t_{0}+\delta\right) .
$$

Since $l<\xi\left(t_{0}\right)$ we can choose (possibly smaller) $\sigma$ and $\delta$ such that

$$
\int_{\beta}^{\beta+\sigma} \frac{b^{\prime}(s)}{\Phi(s)+c s-(\Phi(\beta)+c \beta)} \mathrm{d} s-\int_{\beta}^{\beta+\sigma} \frac{b^{\prime}(s)}{\Phi(s)-c s-(\Phi(\beta)-c \beta)} \mathrm{d} s+2 c \delta<\xi\left(t_{0}\right)-l .
$$

Note that the integrals in the previous inequality are finite thanks to assumption $(16)$. Next, define implicitly the function $U=U(x, t)$ for $t<t_{0}+\delta$ by

$$
\begin{cases}\int_{\beta}^{U} \frac{b^{\prime}(s)}{\Phi(s)+c s-(\Phi(\beta)+c \beta)} \mathrm{d} s=x-x_{1}+c\left(t-t_{0}\right) & \text { if } x \geqslant x_{1}-c\left(t-t_{0}\right), \\ U=\beta \quad \text { if } x_{0}+c\left(t-t_{0}\right)<x<x_{1}-c\left(t-t_{0}\right), & \\ \int_{\beta}^{U} \frac{b^{\prime}(s)}{\Phi(s)-c s-(\Phi(\beta)-c \beta)} \mathrm{d} s=x-x_{0}-c\left(t-t_{0}\right) & \text { if } x<x_{0}+c\left(t-t_{0}\right),\end{cases}
$$


where

$$
\begin{aligned}
& x_{1}=\xi\left(t_{0}\right)-\int_{\beta}^{\beta+\sigma} \frac{b^{\prime}(s)}{\Phi(s)+c s-(\Phi(\beta)+c \beta)} \mathrm{d} s, \\
& x_{0}=l-\int_{\beta}^{\beta+\sigma} \frac{b^{\prime}(s)}{\Phi(s)-c s-(\Phi(\beta)-c \beta)} \mathrm{d} s .
\end{aligned}
$$

Since

$$
\begin{aligned}
& \int_{\beta}^{U\left(\xi\left(t_{0}\right), t\right)} \frac{b^{\prime}(s)}{\Phi(s)+c s-(\Phi(\beta)+c \beta)} \mathrm{d} s=\xi\left(t_{0}\right)-x_{1}+c\left(t-t_{0}\right) \\
& \geqslant \xi\left(t_{0}\right)-x_{1}=\int_{\beta}^{\beta+\sigma} \frac{b^{\prime}(s)}{\Phi(s)+c s-(\Phi(\beta)+c \beta)} \mathrm{d} s,
\end{aligned}
$$

it follows that $U\left(\xi\left(t_{0}\right), t\right) \geqslant \beta+\sigma \geqslant u\left(\xi\left(t_{0}\right), t\right)$ for every $t \in\left[t_{0}, t_{0}+\delta\right)$. Similarly $U(l, t) \geqslant$ $\beta+\sigma \geqslant u(l, t)$ for every $t \in\left[t_{0}, t_{0}+\delta\right)$. Finally, by construction $U\left(x, t_{0}\right) \geqslant \beta=u\left(x, t_{0}\right)$. Applying the comparison principle in the domain $\left(l, \xi\left(t_{0}\right)\right) \times\left[t_{0}, t_{0}+\delta\right)$, we deduce that

$$
u(x, t) \leqslant \beta \quad \text { if } x_{0}+c\left(t-t_{0}\right)<x<x_{1}-c\left(t-t_{0}\right) .
$$

In particular we have

$$
u(x, t) \leqslant \beta \quad \forall(x, t) \text { such that } x_{0}+c \delta<x<x_{1}-c \delta, t_{0} \leqslant t<t_{0}+\delta .
$$

Thanks to (17), this range of values is not empty, hence for every $t \in\left[t_{0}, t_{0}+\delta\right)$ there is an interval $I \subset(\xi(t), \zeta(t))$ such that $u \leqslant \beta$ in $I$. This contradicts the definition of $\xi(t)$, thus $\lim _{t \rightarrow t_{0}^{+}} \xi(t)=$ $\xi\left(t_{0}\right)$.

REMARK 4.5 The proofs of Propositions 4.1, 4.2 and 4.3 still hold if $b$ is only Hölder continuous. In particular the necessary and sufficient conditions of Proposition 4.3 apply to cases of fast diffusion.

\section{REFERENCES}

1. Aregba-Driollet, D., Natalini, R., \& TAng, S. Q. Diffusive kinetic explicit schemes for nonlinear degenerate parabolic systems. Hyperbolic Problems: Theory, Numerics, Applications, Vols. I, II (Magdeburg, 2000), Internat. Ser. Numer. Math. 140, 141, Birkhäuser, Basel (2001), 49-58.

2. Aronson, D. G., Caffarelli, L. A., \& VÁzquez, J. L. Interfaces with a corner point in onedimensional porous medium flow. Comm. Pure Appl. Math. 38 (1985), 375-404. Zbl 0544.35058 MR 0792397

3. Berger, A. E., \& Rogers, J. C. W. Some properties of the nonlinear semigroup for the problem $u_{t}=\Delta f(u)$. Nonlinear Anal. 8 (1984), 909-939. Zbl 0557.35129 MR 0753767

4. Bertsch, M., \& Hilhorst, D. The interface between regions where $u<0$ and $u>0$ in the porous medium equation. Appl. Anal. 41 (1991), 111-130. Zbl 0675.76094 MR 1103850

5. Bertsch, M., \& Klaver, M. H. A. The Stefan problem with mushy regions: differentiability of the interfaces. Ann. Mat. Pura Appl. (4) 166 (1994), 27-61. Zbl 0871.35112 MR 1313798

6. Bertsch, M., \& KlaVer, M. H. A. The Stefan problem with mushy regions: continuity of the interface. Proc. Roy. Soc. Edinburgh Sect. A 112 (1989), 33-52. Zbl 0686.35109 MR 1007536 
7. Bertsch, M., De Mottoni, P., \& Peletier, L. A. Degenerate diffusion and the Stefan problem. Nonlinear Anal. 8 (1984), 1311-1336. Zbl 0562.35049 MR 0764915

8. Bouchut, F., Guarguaglini, F. R., \& Natalini, R. Diffusive BGK approximations for nonlinear multidimensional parabolic equations. Indiana Univ. Math. J. 49 (2000), 723-749. Zbl 0964.35011 MR 1793689

9. BÜRGER, R., EVJE, S., \& KARLSEN, K. H. On strongly degenerate convection-diffusion problems modeling sedimentation-consolidation processes. J. Math. Anal. Appl. 247 (2000), 517-556. Zbl 0961.35078 MR 1769093

10. BÜRGER, R., \& KARLSEN, K. H. A strongly degenerate convection-diffusion problem modeling centrifugation of flocculated suspensions. Hyperbolic Problems: Theory, Numerics, Applications, Vols. I, II (Magdeburg, 2000), Internat. Ser. Numer. Math. 140, 141, Birkhäuser, Basel (2001), 207-216. MR 1882921

11. Bustos, M. C., Concha, F., Bürger, R., \& Tory, E. Sedimentation and Thickening. Phenomenological Foundation and Mathematical Theory. Math. Modelling Theory Appl. 8, Kluwer, Dordrecht (1999). Zbl 0936.76001 MR 1747460 MR 0764915

12. CAfFarelli, L. A., \& Friedman, A. Regularity of the free boundary of a gas flow in an $n$-dimensional porous medium. Indiana Univ. Math. J. 29 (1980), 361-391. Zbl 0439.76085 MR 0570687

13. Caffarelli, L. A., VÁzquez, J. L., \& Wolanski, N. I. Lipschitz continuity of solutions and interfaces of the $N$-dimensional porous medium equation. Indiana Univ. Math. J. 36 (1987), 373-401. Zbl 0644.35058 MR 0891781

14. Carrillo, J. Entropy solutions for nonlinear degenerate problems. Arch. Ration. Mech. Anal. 147 (1999), 269-361. Zbl 0935.35056 MR 1709116

15. Daskalopoulos, P., \& Hamilton, R. The free boundary for the $n$-dimensional porous medium equation. Int. Math. Res. Not. 1997, no. 17, 817-831. Zbl 0886.35114 MR 1474840

16. DiBenedetto, E., \& Vespri, V. On the singular equation $\beta(u)_{t}=\Delta u$. Arch. Ration. Mech. Anal. 132 (1995), 247-309 Zbl 0849.35060 MR 1365831

17. Evje, S., \& KARLSEn, K. H. Monotone difference approximations of BV solutions to degenerate convection-diffusion equations. SIAM J. Numer. Anal. 37 (2000), 1838-1860 Zbl 0985.65100 MR 1766850

18. Eymard, R., Gallouët, T., Herbin, R., \& Michel, A. Convergence of a finite volume scheme for nonlinear degenerate parabolic equations. Numer. Math. 92 (2002), 41-82. Zbl 1005.65099 MR 1917365

19. Gagneux, G., \& Madaune-Tort, M. Analyse mathématique de modèles nonlinéaires de l'ingégnerie pétrolière. Mathématiques \& Applications 22, Springer, Berlin (1996). Zbl 0842.35126 MR 1616513

20. Gilding, B. H. The occurrence of interfaces in nonlinear diffusion-advection processes. Arch. Ration. Mech. Anal. 100 (1988), 243-263. Zbl 0672.76094 MR 0918796

21. Götz, I. G., \& Zaltzman, B. Some criteria for the disappearance of the mushy region in the Stefan problem. Quart. Appl. Math. 53 (1995), 657-671. Zbl 0842.35139 MR 1359501

22. Guarguaglini, F. R., Milisic, V., \& Terracina, A. A discrete BGK approximation for strongly degenerate parabolic problems with boundary conditions. J. Differential Equations 202 (2004), 183-207. Zbl 1058.35125 MR 2068438

23. Karlsen, K. H., Risebro, N. H., \& Towers, J. D. On a nonlinear degenerate parabolic transportdiffusion equation with a discontinuous coefficient. Electron. J. Differential Equations 2002, no. 93, 23 pp. Zbl 1015.35049 MR 1938389

24. Knerr, B. F. The porous medium equation in one dimension. Trans. Amer. Math. Soc. 234 (1977), 381-415. Zbl 0365.35030 MR 0492856

25. KRUŽKov, S. N. First order quasilinear equations in several independent variables. Math. USSR-Sb. 10 (1970), 217-243. 
26. MARCATI, P. Weak solutions to a nonlinear partial differential equation of mixed type. Differential Integral Equations 9 (1996), 827-848. Zbl 0870.35058 MR 1401440

27. Mascia, C., Porretta, A., \& Terracina, A. Nonhomogeneous Dirichlet problems for degenerate parabolic-hyperbolic equation. Arch. Ration. Mech. Anal. 163 (2002), 87-124 Zbl 1027.35081 MR 1911095

28. Matano, H. Nonincrease of the lap-number of a solution for a one-dimensional semilinear parabolic equation. J. Fac. Sci. Univ. Tokyo Sect. IA Math. 29 (1982), 401-441. Zbl 0496.35011 MR 0672070

29. Michel, A., \& Vovelle, J. Entropy formulation for parabolic degenerate equations with general Dirichlet boundary conditions and application to the convergence of FV methods. SIAM J. Numer. Anal. 41 (2003), 2262-2293. Zbl 1058.35127 MR 2034615 